\title{
The Bifunctional Glyceryl Transferase/Phosphatase OzmB Belonging to the HAD Superfamily That Diverts 1,3-Bisphosphoglycerate into Polyketide Biosynthesis
}

\author{
Pieter C. Dorrestein ${ }^{1}$, Steven G. Van Lanen ${ }^{2}$, Wenli $\mathrm{Li}^{2}$, Chunhua Zhao ${ }^{5}$, Zixin Deng ${ }^{5}$, Ben Shen ${ }^{2,3,4, *}$, \\ Neil L. Kelleher ${ }^{1, *}$. \\ ${ }^{1}$ Department of Chemistry, University of Illinois, 600 South Mathews Avenue, Urbana, IL 6180, ${ }^{2}$ Division of \\ Pharmaceutical Sciences, ${ }^{3}$ University of Wisconsin National Cooperative Drug Discovery Group, and \\ ${ }^{4}$ Department of Chemistry, University of Wisconsin-Madison, Madison, Wisconsin 53705, USA. ${ }^{5}$ Laboratory \\ of Microbial Metabolism and School of Life Science and Biotechnology, Shanghai Jiaotong University, \\ Shanghai 200030, China \\ kelleher@scs.uiuc.edu and bshen@pharmacy.wisc.edu
}

\section{Supporting Information}

Chemicals: D-3-phosphoglycerate was from Fluka (Milwaukee, WI). ATP and D-3phosphokinase was purchased from Sigma-Aldrich (St. Louis, MO). Trypsin was purchased from Promega (Madison, WI). dNTPs were from Invitrogen (Carlsbad, CA). Restriction enzymes were from New England Biolabs (Beverly, MA). Oligonucleotides were obtained from University of Wisconsin-Madison Biotechnology Center (Madison, WI).

Cloning of ttmD and ozmB. The genes for $t$ tmD and ozmB were amplified by PCR using pBS6008 ${ }^{1}$ or pJTU $1078^{2}$ respectively, as a template and Expand Long Template PCR System from Roche (Indianapolis, IN). Reactions were performed following manufacture's protocol with 5\% DMSO added and the following primers: $t$ tmD 5'(forward) 5 '

GGTATTGAGGGTCGCATGAAGACATCCTCCGAGCC-3'/(reverse) 5' AGAGGAGAGTTAGAGCCTCACCGCTCGGCTTCCCG-3'; and ozmB (forward) 5' GACGACGACAAGATGACCGAAGCACGGCCGAC-3'/(reverse) 5' GAGGAGAAGCCCGGTTCACTCGGCGTGCGCCCAC-3'. Purified PCR product was inserted into pET-30 Xa $(t t m D)$ or pRSF-Ek $(o z m B)$ using ligation-independent cloning as described by Novagen (Madison, WI), affording pBS6013 (for $t$ tmD) and pBS7001 (for ozmB), respectively, and sequenced to confirm PCR fidelity. These expression constructs, upon introduction into $E$. coli, should result in the production of TtmD and OzmB as N-His ${ }_{6}$-tagged fusion proteins, respectively.

Mutagenesis to generate ozmB(D14V). The Quikchange site-directed mutagenesis protocol (Stratagene, La Jolla, CA) was used to introduce mutations into putative active site residue Asp14 of OzmB. The D14A mutation was introduced into $o z m B$ with the following primer pair: (forward) 5'-ACGATCAAATGCCTGGTCTGGGCCCTGGACAACACGCTGTGGC3'/(reverse) 5'-GCCACAGCGTGTTGTCCAGGGCCCAGACCAGGCATTTGATCGT-3' (with 
the Ala codon underlined). Successful reaction mixtures consisted of $50 \mathrm{ng}$ of pBS7001 as template DNA, each primer at $300 \mathrm{nM}, 500 \mu \mathrm{M}$ dNTPs, 5\% (v/v) DMSO, $1 \times$ buffer, and $2.5 \mathrm{U}$ of cloned Expand Long Template polymerase in a final volume of $50 \mu \mathrm{L}$. The PCR program was as follows: initial denaturing at $96{ }^{\circ} \mathrm{C}$ for $2 \mathrm{~min}$, followed by 20 cycles at $96{ }^{\circ} \mathrm{C}$ for $10 \mathrm{~s}, 56{ }^{\circ} \mathrm{C}$ for $30 \mathrm{~s}$, and $68{ }^{\circ} \mathrm{C}$ for $3 \mathrm{~min}$, and completed by an additional $7 \mathrm{~min}$ at $68^{\circ} \mathrm{C}$. Upon completion, 10 units of $D p n I$ was added directly to the PCR mixture and digested at $37{ }^{\circ} \mathrm{C}$ for $1 \mathrm{~h}$.. The mixture $(1 \mu \mathrm{L})$ was directly transformed into $E$. coli DH5 $\alpha$ and plated on LB supplemented with $100 \mu \mathrm{g} / \mathrm{mL}$ kanamycin. The $o z m B(D 14 A)$ mutant construct was confirmed by sequencing the full ozmB coding region to give pBS7002.

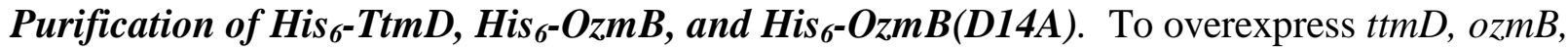
and $o z m B(D 14 A)$ in $E$. coli, pBS6013, pBS7001, and pBS7002 were introduced into E. coli BL21(DE3), respectively, and the resultant recombinants were cultured under standard conditions. Cultures of $500 \mathrm{~mL} \mathrm{LB}$ in $2.5 \mathrm{~L}$ flasks were grown at $18{ }^{\circ} \mathrm{C}$ until $\mathrm{OD}_{600}$ reached 0.5 ( 10 hours) and induced with $0.1 \mathrm{mM}$ IPTG. Cells continued to grow at $18^{\circ} \mathrm{C}$ for 14 hours before harvesting. Affinity purification was performed using Ni-NTA agarose as described by Qiagen (Valencia, CA), and protein was desalted using PD-10 columns (Amersham Biosciences Corp., Piscataway, NJ). Protein purity was assessed as $>90 \%$ by $12 \%$ acrylamide SDS-PAGE, and His $_{6}$-tagged fusion proteins were utilized without further modifications.

Generation of glyceryl-S-OzmB. To prepare glyceryl-S-OzmB, $2 \mu \mathrm{L}$ of OzmB (100 mg/mL), 2 $\mu \mathrm{L}$ of D-3-phosphoglycerate (28 mM), $10 \mu \mathrm{L}$ of ATP $(1.4 \mathrm{mM}), 1 \mu \mathrm{L}$ of $\mathrm{MgCl}_{2}(800 \mathrm{mM}), 2 \mu \mathrm{L}$ of D-3-diphosphoglycerate-kinase (21.4 units) and $50 \mu \mathrm{L}$ of $50 \mathrm{mM}$ Tris, $1 \mathrm{mM}$ TCEP, $\mathrm{pH} 7.6$ were combined. The reaction was allowed to proceed for $5 \mathrm{~min}$ before $50 \mu \mathrm{L}$ of $10 \%$ formic acid was added. The reaction mixture, including precipitate, was loaded onto an HPLC column then separated on a water-acetonitrile gradient. The column used for separations was a Jupiter $5 \mu \mathrm{C} 4$ $300 \AA$ column from Phenomenex (Torrance, CA). The gradient used was: Solvent A is water with $0.1 \%$ TFA and solvent $\mathrm{B}$ is acetonitrile with $0.1 \%$ TFA. The gradient used for the purification was $0-10 \mathrm{~min} 10 \% \mathrm{~B}, 10-12.5 \mathrm{~min} 10-30 \% \mathrm{~B}, 12.5-25 \mathrm{~min} 30-60 \% \mathrm{~B}, 25-30 \mathrm{~min}$ $60-90 \%$ B. It is important to wash the column several times by repeatedly changing from $90 \% \mathrm{~B}$ and back to $30 \%$ B to avoid carry over from the previous samples. As controls D-3phosphoglycerate, ATP, or the kinase were omitted.

Generation of glyceryl-S-TtmD. To prepare holo-TtmD, $80 \mu \mathrm{L}$ of apo-TtmD $(18 \mathrm{mg} / \mathrm{mL})$ was incubated with $40 \mu \mathrm{L}$ of CoA $(3.2 \mathrm{mM}), 1 \mu \mathrm{L}$ of $\mathrm{MgCl}_{2}$, and $4 \mu \mathrm{L}$ of Sfp $(1.2 \mathrm{mg} / \mathrm{mL})$. After incubation at $37{ }^{\circ} \mathrm{C}$ for $60 \mathrm{~min}$. a portion $(30 \mu \mathrm{L})$ of the reaction mixture was added to a reaction containing glyceryl-S-OzmB as described above and allowed to incubate at $37{ }^{\circ} \mathrm{C}$ for 5 min before $50 \mu \mathrm{l}$ of $10 \%$ formic acid was added. The reaction mixture, including precipitate, was loaded onto an HPLC column then separated on a water-acetonitrile gradient as described above. As controls OzmB, D-3-phosphoglycerate or the kinase were omitted.

Generation of 3-phosphoglyceryl-S-OzmB(D14V). To prepare 3-phosphoglyceryl-SOzmB(D14V), $10 \mu \mathrm{L}$ of OzmB(D14V) $(17 \mathrm{mg} / \mathrm{mL}), 2 \mu \mathrm{L}$ of D-3-phosphoglycerate $(28 \mathrm{mM}), 10$ $\mu \mathrm{L}$ of ATP $(1.4 \mathrm{mM}), 1 \mu \mathrm{L}$ of $\mathrm{MgCl}_{2}(800 \mathrm{mM}), 2 \mu \mathrm{L}$ of D-3-diphosphoglycerate kinase $(21.4$ units) and $50 \mu \mathrm{L}$ of $50 \mathrm{mM}$ Tris, $1 \mathrm{mM}$ TCEP, $\mathrm{pH} 7.6$ were mixed. The reaction was allowed to proceed at $37^{\circ} \mathrm{C}$ for $5 \mathrm{~min}$ before $50 \mu \mathrm{L}$ of $10 \%$ formic acid was added. The reaction mixture, 
including precipitate, was loaded onto an HPLC column then separated on a water-acetonitrile gradient (described above). As controls D-3-phosphoglycerate, ATP, or the kinase were omitted.

Trypsin digestion of glyceryl-S-OzmB. $200 \mu \mathrm{L}$ of glyceryl-S-OzmB (prepared identically as described above) was incubated with 80 units of sequence grade trypsin and $10 \mu \mathrm{L}$ of $1 \mathrm{M}$ Tris,

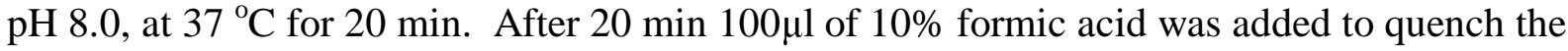
reaction. The reaction mixture was then separated by HPLC on a water-acetonitrile gradient (table 2?). The fraction from 19 to 20 min contained the glyceryl-S-OzmB active site.

Electrospray Fourier tranform mass spectrometry. HPLC fractions containing the active sites prepared as described above were redissolved in $200 \mu \mathrm{L}$ of $49 \%$ methanol and $1 \%$ formic acid and analyzed by ESI-FTMS. For mass spectrometric analysis, a custom 8.5 T ESI-FTMS mass spectrometer was used, which was equipped with a front-end quadrupole. ${ }^{3}$ The samples were introduced into the FTMS using a NanoMate 100 for automated nanospray (Advion Biosciences, Ithaca, NY). Typically $500 \mathrm{~ms}$ ion accumulation per scan was used and 50-200 scans were acquired per spectrum. The instrument was externally calibrated using ubiquitin, $8560.65 \mathrm{Da}$ monoisotopic $\mathrm{M}_{\mathrm{r}}$ value (Sigma). For the calculation of the masses of the proteins, the MIDAS analysis data-station was used. ${ }^{4}$ IRMPD was accomplished by irradiating with a $\mathrm{CO}_{2}$ laser for 80-300 ms and CAD was achieved with a -10 to $-25 \mathrm{~V}$.

\section{References}

(1) Li, W.; Ju, J.; Osada, H.; Shen, B. J. Bacteriol. 2006, 188, 4148-4152.

(2) Zhao, C.; Ju, J.; Christenson, S.D.; Smith, W.C.; Song, D.; Zhou, X.; Shen, B.; Deng, Z. J. Bacteriol. 2006, 188, 4142-4147.

(3) Patrie, S.M.; Charlebois, J.P.; Whipple, D.; Kelleher, N.L.; Hendrickson, C.L.; Quinn, J.P.; Marshall, A.G.; Mukhopadhyay, B. J. Am. Soc. Mass Spectrom. 2004, 15, 10991108.

(4) Senko, M.W.; Canterbury, J.D.; Guan, S.; Marshall, A.G. Rapid Commun. Mass Spectrom. 1996, 10, 1839-1844. 


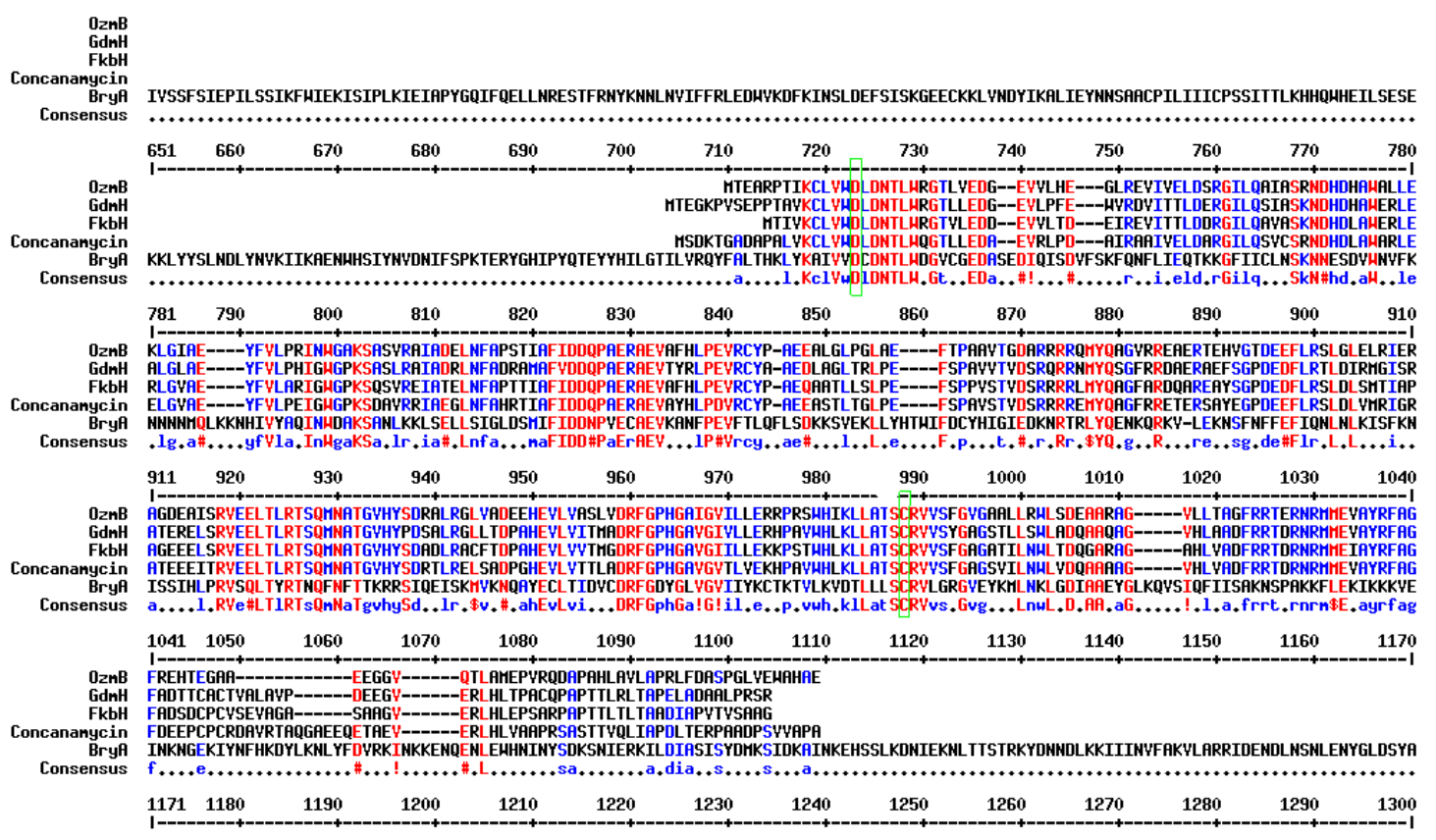

Figure S1. Alignment of HAD containing domains or proteins from the oxazolomycin (OzmB, ABA39082), geldanamycin (GdmH, AAO06922), FK520 (FkbH, AAF86387), Concanamycin (unknown, AAZ94395) and the partial sequence of bryostatin (BryA, AAT48869). Given in parentheses are protein accession numbers. The conserved $\mathrm{D}$ and $\mathrm{C}$ are indicated by a green box. 


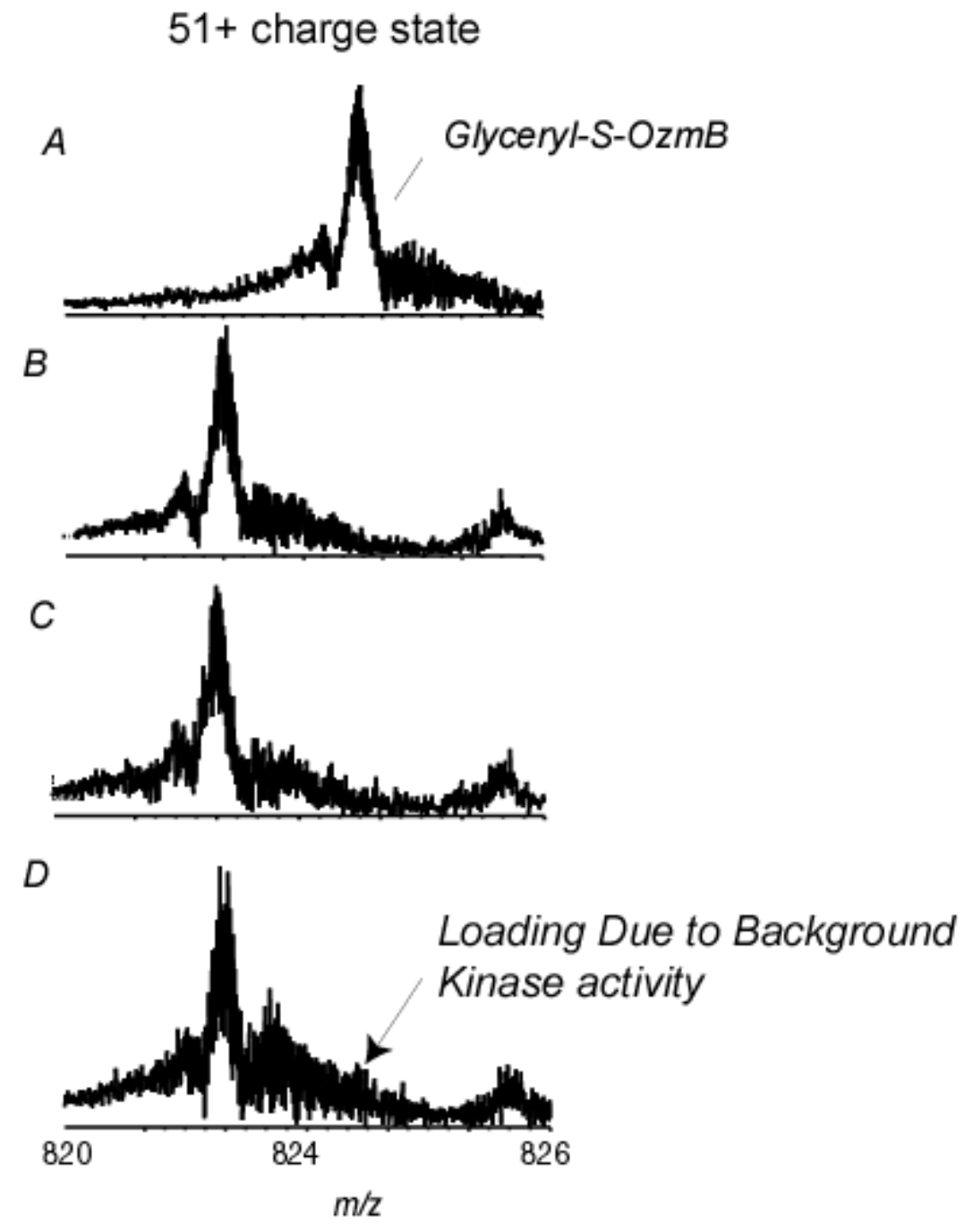

Figure S2. The formation of glyceryl-S-OzmB: (A) OzmB, ATP, D-3-phosphoglycerate, D-3phosphoglycerate kinase; (B) same as (A) but D-3-phosphoglycerate omitted; (C) same as (A) but ATP omitted; and (D) same as (A) but D-3-phosphoglycerate kinase omitted. 


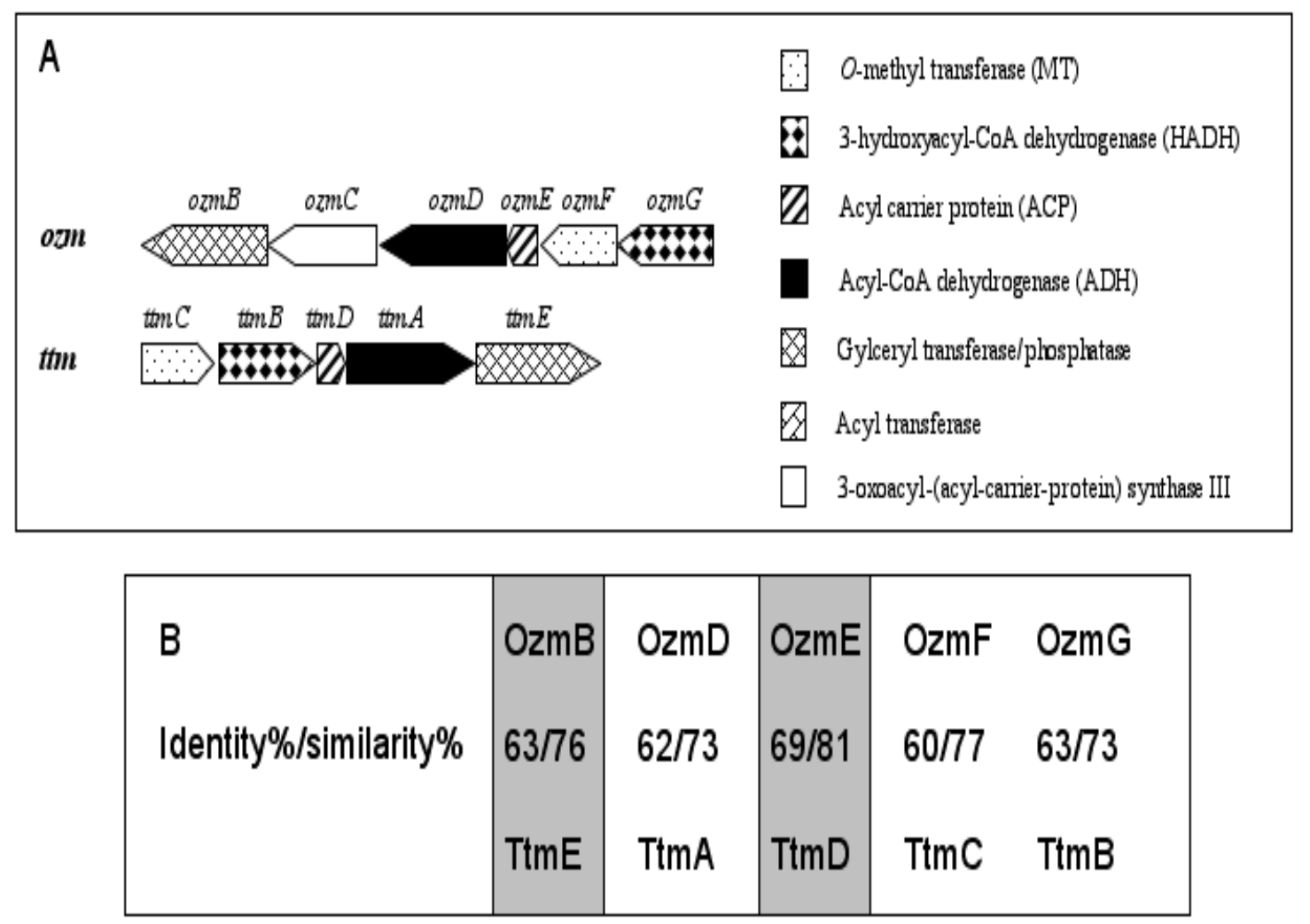

Figure S3. Comparison of (A) the methoxymalonyl-ACP biosynthetic locus for oxazolomycin (ozm) from S. albus ${ }^{2}$ and for tautomycin (ttm) from S. spiroverticillatus ${ }^{1}$ and of (B) amino acid sequences of the homologs within these loci. The two proteins studied in this paper are shaded. 


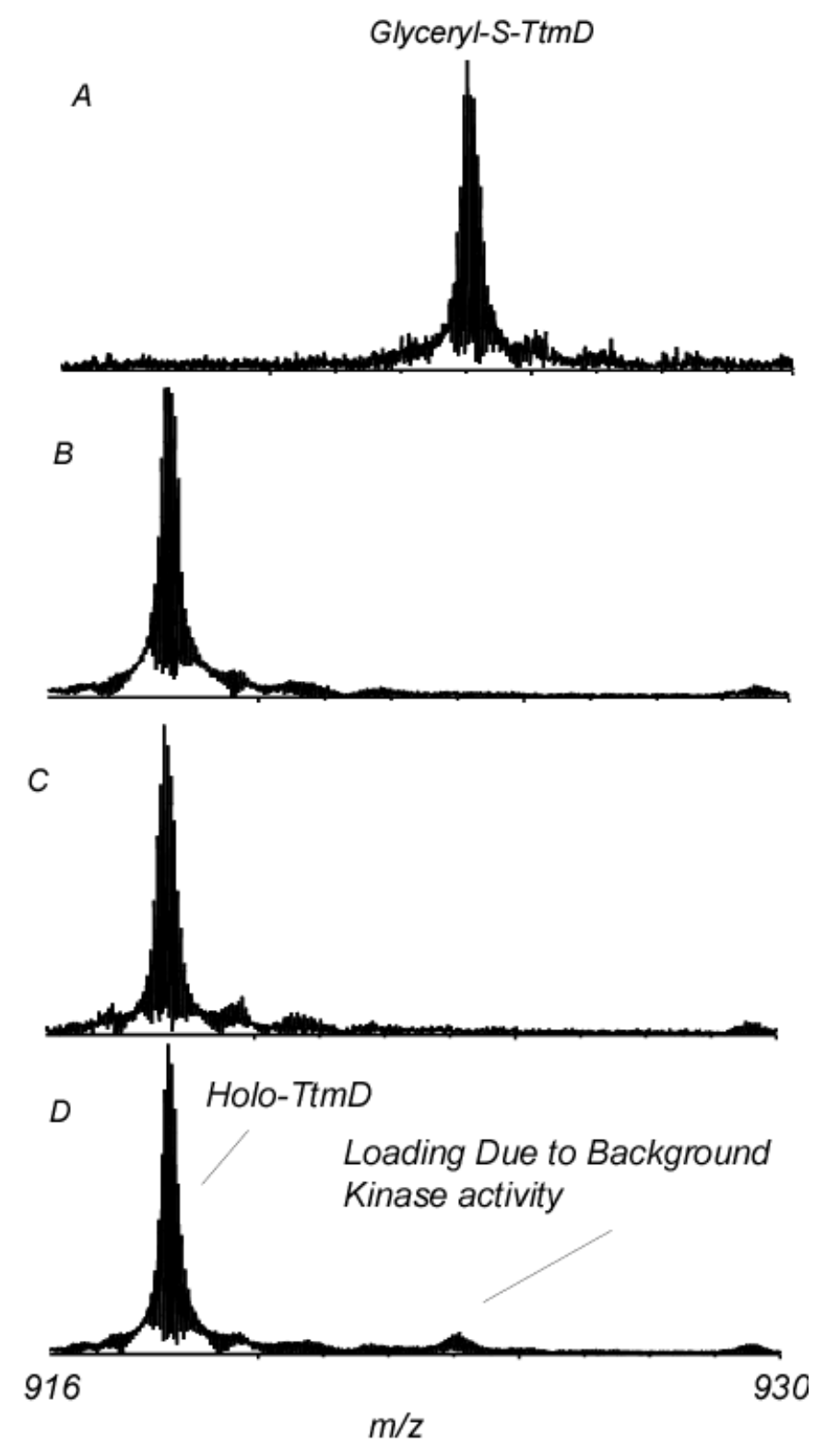

Figure S4. Transfer of glycerate from glyceryl-S-OzmB to TtmD: (A) holo-TtmD, ATP, OzmB, D-3-phosphoplycerate, D-3-phosphoglycerate kinase; (B) same as (A) but D-3-phosphoglycerate omitted; (C) same as (A) but OzmB omitted; and (D) same as (A) but D-3-phosphoglycerate kinase omitted. The ions shown are the $16+$ charge state. 


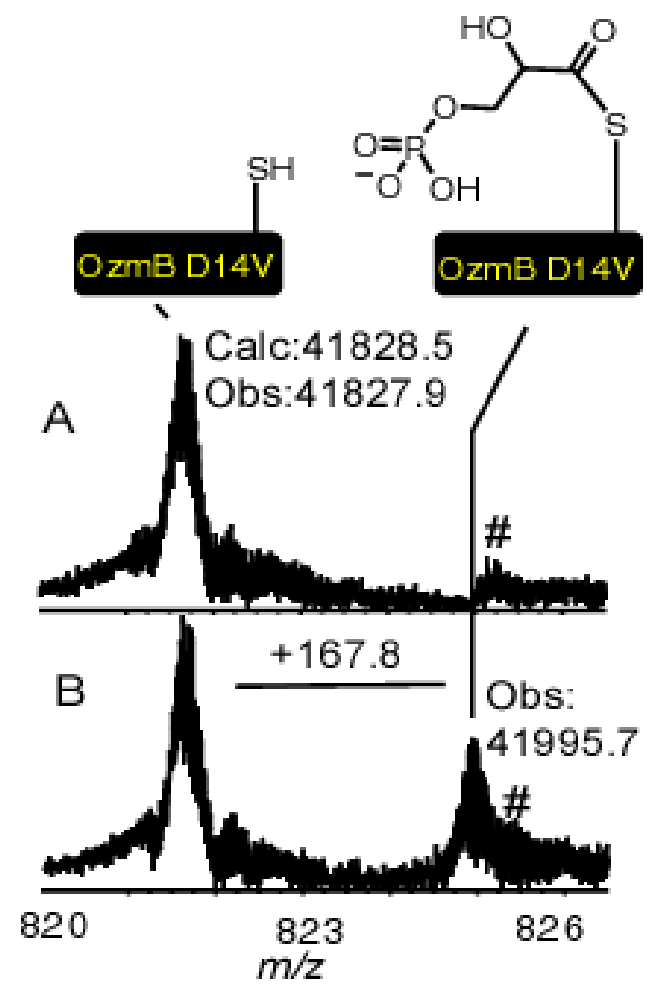

Figure S5. The formation of D-3-phosphoglyceryl-S-OzmB: (A) OzmB(D14V) and (B) OzmB(D14V) incubated with ATP, D-3-phosphoglycerate, and D-3-phosphoglycerate kinase. 